\title{
Feasibility Study For In-Vehicle Detection of Severe Cardiac Events
}

\author{
Kelsey Horan ${ }^{1)}$ Reza Soroushmehr ${ }^{1)}$ Pujitha Gunaratne ${ }^{2)}$ Kazutoshi Ebe $^{3)}$ Kayvan Najarian ${ }^{1)}$ \\ 1) Michigan Center for Integrative Research in Critical Care, University of Michigan, Ann Arbor \\ Building 10-109A, North Campus Research Complex, 2800 Plymouth Road \\ Ann Arbor, Michigan, 48109, U.S.A. \\ (E-mail: kelhoran@med.umich.edu) \\ 1) Department of Computational Medicine and Bioinformatics, University of Michigan, Ann Arbor, MI, 48109, USA \\ (E-mail: \{ssoroush,kayvan\}@med.umich.edu) \\ 2) Toyota Motor North America, Inc. \\ 1555 Woodridge Ave, Ann Arbor, MI 48105 (Email: pujitha.gunaratne@toyota.com) \\ 3) Toyota Central R\&D Labs., Inc. Human Science Research-Domain \\ 41-1, Yokomichi, Nagakute, Aichi 480-1192,Japan (E-mail: kzebe@mosk.tytlabs.co.jp)
}

Received on October 13, 2017

\begin{abstract}
Cardiovascular diseases are highly prevalent and fatal medical conditions cause many adult deaths annually. The occurrence of a debilitating cardiac condition while driving could suddenly render a driver unable to safely operate a vehicle, including bringing a vehicle to a stop in order to request medical assistance. Currently, many people are at risk of suffering from conditions such as a heart attack or arrhythmia. We conduct a feasibility study to determine the practicality of developing an in-vehicle severe cardiac event detection system, integrated with a driver monitoring system, to detect or predict these events. We provide background information related to the primary diagnostic criteria and evaluation of the Electrocardiogram (ECG). A preliminary noise analysis is conducted to compare the ECG noise of in-vehicle and in-lab signals. Ultimately, we determine that developing a driver monitoring system capable of determining the driver's cardiac health is completely feasible.
\end{abstract}

KEY WORDS: human engineering, physiological monitoring, medical event detection, autonomous driving technology \& driver assistance systems, vehicular sensors and environment perception, ITS and ICT for safety applications[C2]

\section{Introduction}

Every year cardiovascular diseases result in a high number of deaths globally. These events include Myocardial Infarction (MI), also known as heart attacks, and a wide array of other arrhythmias such as Supraventricular Tachycardia, Ventricular Tachycardia, Bradycardia, Atrial Fibrillation, and Ventricular Fibrillation. MI, as well as the other listed complications, present uniquely in the Electrocardiogram (ECG) signal, which records electrical activity throughout the regions of the heart. Recent overwhelming advances in mobile monitoring systems for ECG, as well as advances in the signal quality provided in these devices, have made possible the convenient collection of data from at-risk patients without impacting mobility or attentiveness. This data can be used in signal processing and machine learning algorithms to assist patients, clinicians, and emergency workers, in recognizing the onset of medical events.

The symptoms of these medical events, such as loss of mobility and syncope, can completely incapacitate a driver. These dangers, coupled with the prevalence of cardiovascular diseases, show that in order to increase the driver safety and decrease accidents it is important to take precautions against the occurrence of in-vehicle cardiac events. Around 5\% of accidents involving commercial vehicles have been attributed to cardiovascular disease in North America ${ }^{(1)}$. The goal of our study is to determine the feasibility of constructing an in-vehicle severe cardiac event detection system that can be incorporated into a Driver Monitoring System (DMS) or an Autonomous Driving System (ADS). In this study we investigate whether the relevant sensors/monitors and algorithms maintain their robustness when adapted to in-vehicle settings.

There are several complications that must be considered to determine the portability of these sensors and algorithms. Firstly, accurate classification of cardiac events requires a high quality signal. Implementing an in-vehicle detection system requires that the in-vehicle noise does not render the ECG signal undetectable, therefore there cannot be significant in-vehicle interference with the typical $5-40 \mathrm{~Hz}$ PQRS complex provided by the ECG. Secondly, there can be an increase in motion artifact due to driver activity. Typical driving motions, such as wheel turning, can also introduce electromyography noise and interfere with the accuracy of detection. Third, choice of monitor is highly important because the monitor must be non-invasive and seamless with the driving task. The monitor can be a small portable monitor such as Holter monitor that is a pre-existing pacemaker and one of the widely available mobile ECG monitors, or incorporated into the vehicle. Fourth, adapting signal processing and machine learning algorithms to in-vehicle requires a diverse dataset. However, to the best of our knowledge there is no sufficient amount of invehicle ECG data available and it yet needs to be compiled. Finally, rigorous training and testing of the detection system must be done to ensure accuracy and avoid false alarms.

Our preliminary in-vehicle noise analysis determines that the noise frequency bands are probably not detrimental to the ECG signal. For example, standard signal processing algorithms such as peak-detection can still be performed on the collected ECG with comparable accuracy. As peak detection is imperative in Heart Rate Variability (HRV) analysis, ensuring the accuracy of such algorithms is essential to severe cardiac event detection. Additionally, we perform the following steps: 1) Removing noise for the type of noise that are unavoidable for ECG collection, 2) Investigating minimal requirements for a mobile ECG monitoring device, 3) Presenting popular and potentially useful databases for training and testing such algorithms. 
In this work we determine that designing an in-vehicle detection system for severe cardiac events is completely feasible. As incorporating an ECG monitoring device into an existing DMS will ensure driver safety, future studies are planned to this end.

\section{Electrocardiograph (ECG)}

According to the Centers for Disease Control and Prevention (CDC), heart disease is the leading cause of death in both men and women in the United States. Every year about 735,000 Americans have a heart attack, and 210,000 of these patients have already had a heart attack. Approximately half of Americans have at least one of the key risk factors for MI such as high blood pressure, high cholesterol level and smoking. This illustrates the importance of early detection of cardiac events.

MI and Ischemia, as well as other severe and debilitating arrhythmias are highly prevalent. The diagnostic criteria for these cardiac events include the quantitative analysis of various cardiovascular parameters as well as a qualitative analysis of the electrocardiograph (ECG) waveform. This analysis is performed by a cardiologist to ensure diagnostic validity. The ECG is typically the gold standard for evaluation and diagnosis of cardiac events. Electrocardiography non-invasively records the electrical activity of the heart over time. Abnormalities and deviation from the standard ECG waveform morphology can indicate that a patient is experiencing a cardiac event with some levels of severity. While an ECG waveform might slightly vary among different subjects, the PQRS complex of a healthy subject is expected to take a particular form.

The ECG monitor includes a various number of sensors, depending on the desired number of leads $(15-, 12-, 10-, 6-, 3-$ lead, etc.), typically placed on the chest. Wearable, portable and non-contact options are available for the ECG, providing various levels of signal quality. Signal processing can be used on the ECG signals to extract features that are indicative of a cardiac event, such as heart rate, heart rate variability, respiratory rate, etc.

Due to the high level ECG monitors and advanced signal processing techniques, there has been plenty of recent works on signal processing and machine learning algorithms for the detection and prediction of these cardiac events, including MI These algorithms serve to filter noise from the raw ECG signal, extract features that are indicative of medical events, and ultimately make a diagnostic recommendation using the ECG signals. Current algorithms work towards either detecting a medical event at onset, predicting a medical event prior to onset or prior to the diagnostic point.

Recent studies show that HRV analysis has been performed on in-vehicle ECG data to detect drowsiness or medical conditions with cardiac manifestations. Yang et al. ${ }^{(2)}$ provide an HRV analysis using Hilbert Huang transform to decompose a signal into Intrinsic Mode Functions. Roy et al. ${ }^{(3)}$ perform ECG analysis on in-vehicle data, alerting drivers to changes in HR that could be indicative of a lack in alertness. Hu et al. ${ }^{(4)}$ show that an adaptive filter can remove the in-vehicle noise on a pulse wave sensor employed to detect driver's drowsiness. HRV analysis is shown to be fairly successful in these situations, although the studies use a small amount of ECG data.

Frameworks for non-contact car-seat ECG collection have been proposed and analyzed ${ }^{(5)}$. The authors calculate features such as HR. Leonhardt and Aleksandrowicz ${ }^{(6)}$ present an in-seat ECG sensor as well. They discuss that the running motor of a vehicle was not a significant source of noise, while the vehicle shock-absorbers, vehicle type, and body movements caused by driving did contribute to noise. Schneider et al. ${ }^{(7)}$ achieve $95.5 \%$ sensitivity and Positive Predictive Value (PPV) of $92 \%$ when comparing signal processing results on data collected by a wearable ECG monitor vs. an in-vehicle seat ECG, suggesting that additional in-vehicle noise is manageable. The analytical discussion mentions that artifacts can be highly correlated with vehicle movement and therefore vehicle motion data should be used to de-noise the ECG signals. Additionally, detection of signal segments that are irreversibly saturated with noise could be handled by a machine learning algorithm, such as Support Vector Machine (SVM) approaches. An algorithm is presented by Wartzek et al. ${ }^{(8)}$ to distinguish ECG intervals contaminated with artifacts that occur between relatively cleaner signal intervals, allowing for a non-contact heart rate estimation on $86 \%$ of test subjects on the highway. Detection increased when clothing restrictions and motion restrictions were placed on the subjects.

The prior works on in-vehicle ECG monitoring show that signal processing is possible. A better understanding of in-vehicle noise will assist in contributing to this goal, further developing these methods, and developing an in-vehicle heart monitoring device capable of detecting and predicting cardiac events.

In summary, according to recent literature simple signal processing methods such as HR and HRV extraction are successful on in-vehicle ECG data. The additional noise for the in-vehicle scenario appears to be motion noise caused by the vehicle, as opposed to electric motors.

We have performed an independent analysis to preliminarily validate these results prior to developing an in-vehicle driver monitoring system for detecting severe cardiac events on a larger scale. Additionally, the analysis provides information about specific frequency bands that are affected most by the vehicle. The results are presented in Section 6.

\section{ECG Noise Types}

The Signal to Noise Ratio (SNR) measures the ratio between the power of a pure signal and that of the noise present in the collected raw signal. An ECG signal consists of low frequencies; a broad estimate of ECG signal frequency range is $0.5 \mathrm{~Hz}-100$ $\mathrm{Hz}$, but typically ECG signals occur below $40 \mathrm{~Hz}$. Formal noise analysis requires an understanding of the types of noise that are applicable in specific situations. Below is a list of noise types found in a raw ECG signal, even in clinical settings $(9,10)$. Broadly, a majority of these noises are attributable to either motion or electrical interference, which are both highly prevalent in-vehicle. A number of noise types that are present in most ECG data follow.

- Powerline Noise is a constant noise caused by powerlines that occurs at $50 \pm 0.2 \mathrm{~Hz}$ or $60 \pm 0.2 \mathrm{~Hz}$, with an amplitude of up to $50 \%$ of full scale deflection (FSD).

- Electrode Noise is an occasional noise occurring at around $60 \mathrm{~Hz}$ that lasts approximately one second, caused by loss of contact between the subject and an electrode. Electrode pop can reach full saturation.

- Motion Noise is caused by electrode movement and patient motion, resulting in impedance changes that manifest as rapid baseline jumps or full saturation segments in the ECG.

- Electromyography (EMG) is interference caused by the electrical activity of other muscles surrounding the heart. This noise can occur from DC to $10,000 \mathrm{~Hz}$ with an amplitude of up to $10 \%$ FSD. 
- Baseline Drift is a constant noise caused by the collection hardware, as well as respiration that occurs at approximately 0.15 to $0.3 \mathrm{~Hz}, 15 \% \mathrm{FSD}$, consistently throughout ECG collection.

- Instrument Noise is caused by the ECG collection devices, and consistently interferes with the collected signal.

- Electro-surgical Noise is caused by additional equipment surrounding the patient, occurring at approximately $100 \mathrm{kHz}-$ $1 \mathrm{MHz}$.

- Digitizing, i.e. quantization, additionally changes raw signals during collection and causes some noise.

Additional continuous sources of noise that should be considered for the in-vehicle scenario are audio frequency (FM radio transmission), radio frequency (wireless and radio transmissions, receivers, industrial scientific and medical equipment, digital processing circuitry), and broadband (cellular mobile telephony). Non-continuous noise, such as electromagnetic impulse, can be caused by electrostatic discharge, lightning, nuclear, powerline, electric motors, electrical ignition systems, and switching actions of digital electronic circuitry. Electromagnetic interference from communication devices was most usually confused by clinicians for atrial fibrillation, ventricular arrhythmias, and pacemaker dysfunction ${ }^{(11)}$. This shows that these types of noises, even clinically, can interfere with the diagnosis of specific medical events.

While the ability to remove all noise from ECG records is limited, a large number of noise removal techniques can be applied to allow for valid diagnostics to be performed on ECG records. We should remove those types of noise from ECG records that help us process the signals comparably to clinical ECGs and maintain the correctness of evaluation by an algorithm.

\section{Monitoring Devices}

In-vehicle ECG monitoring requires specific and advanced medical hardware. A mobile ECG monitoring device that upholds clinical standards is the most optimal choice for collecting data invehicle. There is clearly a hardware complexity difference between the ideal monitoring device in a clinical setting versus the ideal device in-vehicle. Clinically, a more complex ECG sensor with a high sampling rate and a large number of channels provides the best record for cardiac function analysis. Due to invehicle complications, clinical standards must be slightly relaxed in order to permit a driver to perform regular movements and activities. Additionally, due to the need for real-time analysis further hardware restrictions are placed on the sensor. Most signal processing and machine learning techniques can be performed on a raw signal that is monitored at the proper frequency. Therefore, in order to process the ECG signal accurately and in real-time it is optimal that a sensor has the following attributes.

The sensor should provide the raw, unprocessed, ECG signal, as well as communicate the signal in real-time via bluetooth or an equivalent method. The sensor should be mobile with a low number of leads, i.e. wires and electrodes, to avoid obstructing driver abilities. In addition, ideally the device would not interfere with the driver's range of motion by having an inconvenient size or sensor placement. The monitoring device should be Food and Drug Administration (FDA) approved, or have equivalent international approval, and collect samples at a rate of $500 \mathrm{~Hz}$ or higher.

When these requirements are satisfied it is possible to perform real-time signal processing and classification of the ECG waveform on a beat-to-beat basis, as is necessary for real-time cardiac event prediction and detection in a DMS.

\section{Databases}

An important aspect of training and testing high level machine learning and signal processing algorithms, such as invehicle detection of cardiac events, is the availability of large volumes of diverse, relevant and quality databases. In order to ensure the correctness and validity of detection models a developer must ensure that the data used is representative of a vast array of subject types with varying medical histories, as well as a vast array of medical events.

In the case of in-vehicle cardiac event detection correctness is incredibly important. If the DMS is required to respond to alerts of cardiac events, it is necessary that the level of false positives is relatively low. Additionally, due to the danger of missing a debilitating cardiac event, false negatives must be low as well.

Many studies perform data collection prior to algorithm development, to obtain data that was collected in a suitable environment while being representative of the research specific conditions. Consequently, there is a low volume of in-vehicle ECG data. Clinically collected ECG data is openly available through various groups such as PhysioNet ${ }^{(12)}$.

The following is a list of databases widely available that are used for benchmarking by many researchers:

The PTB Diagnostic ECG Dataset ${ }^{(13)}$ contains ECG records, static data and medical history, as well as annotations of clinical diagnosis, from patients presenting with heart failure, MI and various arrhythmias. The 549 ECG records were collected from 290 patients by the Department of Cardiology of the University Clinic Benjamin Franklin, and were digitized by the National Metrology Institute of Germany. Each ECG record contains 15leads, $12+3$ Frank Leads, available at up to $10 \mathrm{kHz}$ sampling rate upon request.

The European ST-T Dataset ${ }^{(14)}$ contains two hour ECG records, as well as annotations of waveform morphology irregularities representing ST and T-wave changes indicative of MI. The 768 episodes are collected from 78 patients by the Institute of Clinical Physiology of the National Research Council, and the Thoraxcenter of Erasmus University. This dataset provides a common benchmarking dataset for MI detection algorithms using ECG and is widely prevalent throughout biomedical signal processing research.

The MIT-BIH Arrhythmia Dataset ${ }^{(15)}$ contains 48 ECG records annotated for various severe arrhythmias of interest as well as annotations for arrhythmia type. The 2-lead, $360 \mathrm{~Hz}$, half hour records were collected from 47 patients at the Beth Israel Deaconess Medical Center, and Massachusetts Institute of Technology. The dataset provides a common benchmarking dataset for arrhythmia detection algorithms using ECG and is used by many studies for algorithm evaluation.

The Long Term ST Dataset ${ }^{(16)}$ contains up to 24-hour long term ECG records as well as annotations for ST deviations. The 86 ECG records were collected by University of Ljubljana, University Medical Center (Ljubljana, Slovenia), CNR Institute for Clinical Physiology at University of Firenze,, Massachusetts Institute of Technology, and the Beth Israel Deaconess Medical Center.

Researchers can supplement datasets with additional records, 
consisting of ECGs that have synthetic in-vehicle noise or retroactively introduced authentic in-vehicle noise. Most importantly, it is advisable, while not very practical, to additionally test algorithms on in-vehicle ECG records of at-risk patients as well as patients who suffer from a cardiac condition while in the vehicle. To completely evaluate the correctness of the algorithm, the experiments must show that the algorithm is capable of real-time driver monitoring. The challenges presented with testing an in-vehicle detection algorithm are directly related to the difficulty of safely collecting this necessary data.

\section{Preliminary Signal Analysis}

One aspect of our feasibility analysis for in-vehicle cardiac event detection is the noise evaluation. Additional in-vehicle noises, such as those mentioned in Section 3, could directly interfere with cardiac frequency bands or completely saturate the record and render the ECG undetectable or un-processable. We performed a preliminary analysis of additional in-vehicle noise to determine the practicality of in-vehicle ECG processing with currently available hardware and algorithms.

For the independent ECG noise assessment we have collected data on subjects both in-vehicle and in-lab. Data was collected under supervision and processed with wavelet transformation. Following this, a statistical t-test was performed on the wavelet coefficients to determine which frequency subbands have a statistically significant difference in coefficient values. Experimental methodology details are outlined below.

Data acquisition was performed with a clinical grade heart monitor, the BodyGuardian sensor made by Preventice Solutions (Rochester, Minnesota, USA). This sensor is a discrete wireless non-intrusive body-worn monitor that captures ECG signals. This sensor could be used for rhythm monitoring, calculation of average heart rate, respiration rate, and activity level. This sensor transmits patient's data including ECG, accelerometer and impedance waveforms automatically to a secure database. In the experiment, 5 minutes of in-lab and 5 minutes of in-vehicle data were collected on four subjects. During in-lab data collection subjects were permitted to perform natural movements while maintaining a comfortable seated position. These natural movements were permitted to introduce noise similar to the natural movements performed by a driver while operating a vehicle. During in-vehicle data collection, the subjects operated a vehicle without any induced distraction, such as music, conversation, and phone calls. All subjects were members of the Wearable Sensing \& Signal Processing Lab at University of Michigan - Dearborn lab. All ECG signals were recorded in similar situations, without allowing for a high amount of environmental variation.

To initially process the data, we first apply a lowpass filter using the Butterworth filter with the order of 2. The frequency response of the $n$th order Butterworth filter can be defined as:

$$
H(\omega)=\frac{1}{\sqrt{1+\left(\frac{\omega}{\omega_{c}}\right)^{2 n}}}
$$

where $\omega_{c}$ is the cutoff frequency. In order to differentiate ECG signals collected in the lab from those collected in vehicles we analyze the ECG signals by applying wavelet packet decomposition on all collected signals. The motivation in using wavelet transformation is that it yields frequency spectrum estimation. Wavelet packet decomposition iteratively constructs a wavelet packet tree, and transforms a signal in the time domain to the frequency domain that can be used for noise analysis. The input is split into approximation and detail coefficients. Similarly, each of these sets of coefficients can be further decomposed. In Fig.1 a two-level signal decomposition is shown.

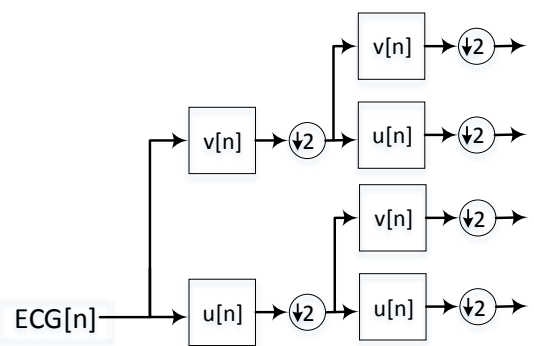

Fig. 1 Decomposing an ECG signal to approximations and details coefficients in a two-level wavelet packet decomposition.

In Fig. 1 it is assumed that $v[n]$ and $u[n]$ are high-pass and lowpass filters respectively. Also, after filtering the signal, downsampling by a factor of 2 is applied. The Daubechies 8 wavelet, "db8" was used to decompose the signal up to 6 levels, to extract coefficients which describe an approximation of the raw ECG signal in the frequency domain. The difference in the wavelet coefficients between in-vehicle and in-lab records for a single patient indicates the noise difference.

We then identify the frequency sub-bands in which invehicle data shows a high deviation from in-lab data. These frequency sub-bands are referred to as the frequency "bands of interest." Following the processing, a paired sample statistical ttest was performed between the wavelet coefficients of the invehicle and in-lab ECG data for each patient. The null hypothesis for the paired sample $t$-test was that the two sets of wavelet coefficients were sampled from the same underlying distribution. We show the t-test outputs as $(h, p)$ where the $h$-value is a boolean, taking the value of 0 , fail to reject the null hypothesis, or 1 , reject the null hypothesis. Also, the $p$ output is the $p$-value for the $t$-test.

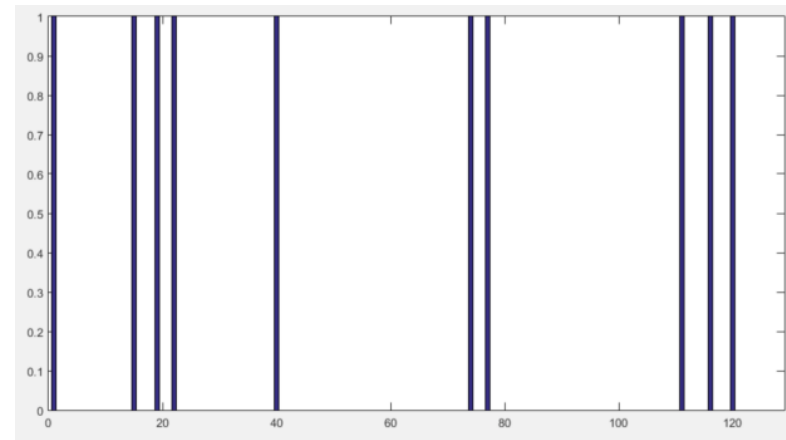

Fig. 2 h-values, Subject One

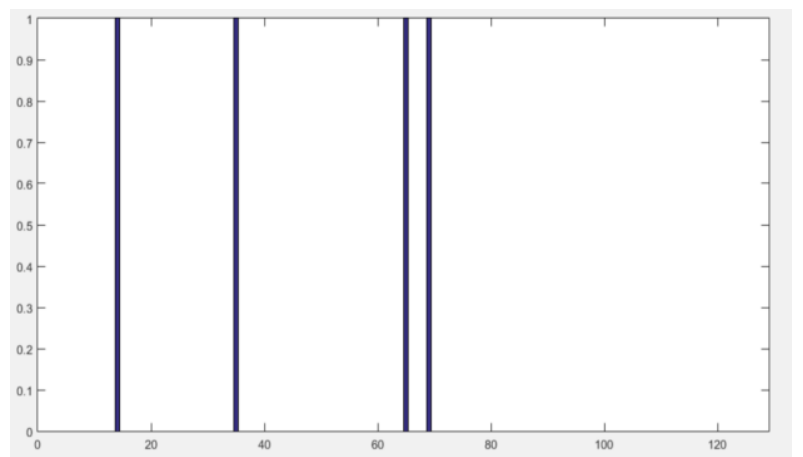

Fig. 3 h-values, Subject Two 


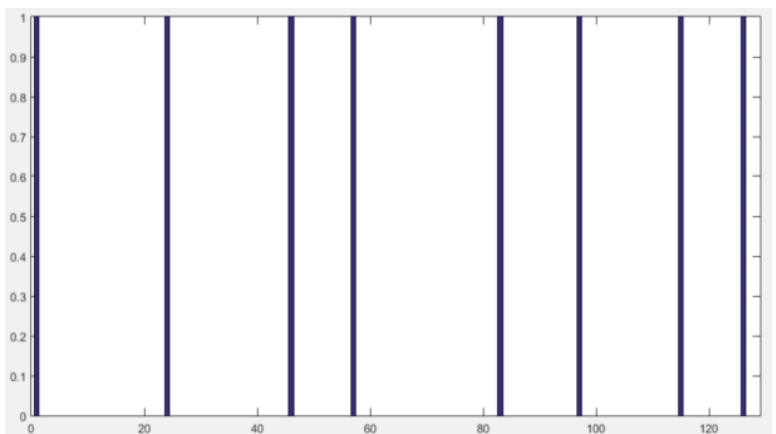

Fig. 4 h-values, Subject Three

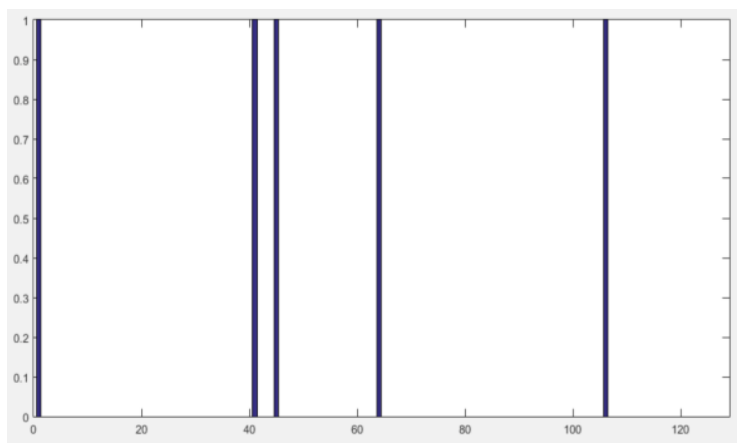

Fig. 5 h-values, Subject Four

Above, in Figures 2 to 5, we plot the $h$ values of the $t$-test against the 128 wavelet coefficients. An $h$-value of 0 indicates that the wavelet coefficients for that set of coefficients did not differ significantly between the in-lab and in-vehicle data. An $h$ value of 1 indicates that the wavelet coefficients were significantly different, detecting a potential frequency band of interest. Tables $1,2,3$ and 4 illustrate the frequency bands of interest for each subject.

Table 1 Subject One Bands of Interest

\begin{tabular}{|l|l|}
\hline $\begin{array}{l}\text { Lower } \\
\text { Bound }\end{array}$ & $\begin{array}{l}\text { Upper } \\
\text { Bound }\end{array}$ \\
\hline \hline $0 \mathrm{~Hz}$ & $1 \mathrm{~Hz}$ \\
\hline $14 \mathrm{~Hz}$ & $15 \mathrm{~Hz}$ \\
\hline $18 \mathrm{~Hz}$ & $19 \mathrm{~Hz}$ \\
\hline $21 \mathrm{~Hz}$ & $22 \mathrm{~Hz}$ \\
\hline $39 \mathrm{~Hz}$ & $40 \mathrm{~Hz}$ \\
\hline $73 \mathrm{~Hz}$ & $74 \mathrm{~Hz}$ \\
\hline $110 \mathrm{~Hz}$ & $111 \mathrm{~Hz}$ \\
\hline $115 \mathrm{~Hz}$ & $116 \mathrm{~Hz}$ \\
\hline $119 \mathrm{~Hz}$ & $120 \mathrm{~Hz}$ \\
\hline
\end{tabular}

Table 2. Subject Two Bands of Interest

\begin{tabular}{|l|l|}
\hline $\begin{array}{l}\text { Lower } \\
\text { Bound }\end{array}$ & $\begin{array}{l}\text { Upper } \\
\text { Bound }\end{array}$ \\
\hline \hline $13 \mathrm{~Hz}$ & $14 \mathrm{~Hz}$ \\
\hline $34 \mathrm{~Hz}$ & $35 \mathrm{~Hz}$ \\
\hline $64 \mathrm{~Hz}$ & $65 \mathrm{~Hz}$ \\
\hline $68 \mathrm{~Hz}$ & $69 \mathrm{~Hz}$ \\
\hline
\end{tabular}

Table 3 Subject Three Bands of Interest

\begin{tabular}{|l|l|}
\hline $\begin{array}{l}\text { Lower } \\
\text { Bound }\end{array}$ & $\begin{array}{l}\text { Upper } \\
\text { Bound }\end{array}$ \\
\hline \hline $0 \mathrm{~Hz}$ & $1 \mathrm{~Hz}$ \\
\hline $23 \mathrm{~Hz}$ & $24 \mathrm{~Hz}$ \\
\hline $45 \mathrm{~Hz}$ & $46 \mathrm{~Hz}$ \\
\hline $56 \mathrm{~Hz}$ & $57 \mathrm{~Hz}$ \\
\hline $82 \mathrm{~Hz}$ & $83 \mathrm{~Hz}$ \\
\hline $96 \mathrm{~Hz}$ & $97 \mathrm{~Hz}$ \\
\hline $114 \mathrm{~Hz}$ & $115 \mathrm{~Hz}$ \\
\hline $125 \mathrm{~Hz}$ & $126 \mathrm{~Hz}$ \\
\hline
\end{tabular}

Table 4 Subject Four Bands of Interest

\begin{tabular}{|l|l|}
\hline $\begin{array}{l}\text { Lower } \\
\text { Bound }\end{array}$ & $\begin{array}{l}\text { Upper } \\
\text { Bound }\end{array}$ \\
\hline \hline $0 \mathrm{~Hz}$ & $1 \mathrm{~Hz}$ \\
\hline $40 \mathrm{~Hz}$ & $41 \mathrm{~Hz}$ \\
\hline $44 \mathrm{~Hz}$ & $45 \mathrm{~Hz}$ \\
\hline $63 \mathrm{~Hz}$ & $64 \mathrm{~Hz}$ \\
\hline $105 \mathrm{~Hz}$ & $106 \mathrm{~Hz}$ \\
\hline
\end{tabular}

The presence of lower frequency bands of interest can be attributed to the fact that standard drift removal was not performed. This shows that the drift patterns of ECG between invehicle and in-lab are different. Performing drift removal on ECG data is well explored and easily handled, resulting in less bands of interest per subject. For instance, median filtering can be applied on ECG signals as a simple approach to remove the baseline drift noise.

These bands of interest are specific frequencies that should be explicitly considered when adapting signal processing methods, such as noise removal, to the in-vehicle scenario. Upon further inspection, it is evident that aside from in-vehicle drift, frequency bands around $20 \mathrm{~Hz}$ and $40 \mathrm{~Hz}$ contain noise variation.

When the large range of frequencies relative to the small number of bands of interest is considered, the noise difference between these two environments is minimal. Figure 6 plots the sum of all $h$ values against the wavelet trees to further highlight the bands of interest. Clearly, the $0-1 \mathrm{~Hz}$ frequency band is a prevalent issue, taking the value of 3 because 3 subjects have this band of interest, reinforcing the need for drift removal. In addition, there appears to be a cluster of frequency bands of interest surrounding $20 \mathrm{~Hz}$ and $40 \mathrm{~Hz}$ that may interfere with a typical ECG signal. However, a larger study will assist in drawing more generalized conclusions. Therefore, the frequency bands shown in this figure might change when we increase the number of subjects.

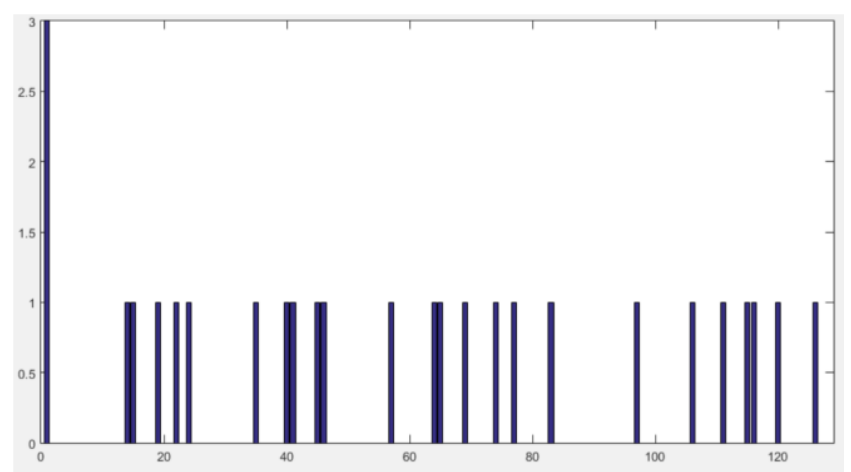

Fig. 6 Summation of $h$-values for all Subjects 
Additionally, peak detection was performed on the in-vehicle and in-lab ECG records. Peak detection and beat detection are important preliminary tasks for beat-to-beat ECG classification. These algorithms ${ }^{(17)}$ can help to detect the QRS complex and ST segment of the ECG, assisting in the morphological analysis of irregular waveforms, for example ST-Elevated MI. Table 5, detailing the False Positive (FP) and False Negative (FN) for each subject. The data used for peak detection was median filtered, normalized, and was processed for drift removal to remove some differences. This preprocessing is standard prior to performing peak detection. A FP corresponds to a peak, i.e. a heartbeat, detected in the absence of a peak, while a FN corresponds to a missed heartbeat. As we can see in the table, within 5 minutes of collected beats the number of false positives and false negatives per subject is minimal. This indicates that certain signal processing methods, and their results, do not vary greatly between the two data collection environments.

Table 5 Peak Detection False Positives and False Negatives

\begin{tabular}{|l|l|l|l|}
\hline Subject & Data Type & FP & FN \\
\hline \hline One & In-Lab & 0 & 1 \\
\hline & In-Vehicle & 0 & 0 \\
\hline \hline Two & In-Lab & 4 & 0 \\
\hline & In-Vehicle & 4 & 0 \\
\hline \hline Three & In-Lab & 0 & 1 \\
\hline & In-Vehicle & 1 & 0 \\
\hline \hline Four & In-Lab & 1 & 0 \\
\hline & In-Vehicle & 1 & 0 \\
\hline
\end{tabular}

Our data collection and analysis showed frequency bands of interest that need to be further investigated during the in-vehicle ECG processing task. A simple peak-detection algorithm shows that, in our scenario, in-vehicle noise is comparable to in-lab noise, in terms of FP and FN.

In-vehicle noise can be approximated and modeled using an accelerometer on ideally both the car and the driver. This additional data could be combined with the raw ECG, allowing for proper noise management and the ability to apply complicated signal processing and machine learning techniques beyond peak detection. Our analysis of in-vehicle data shows that, except for a few frequency sub-bands of interest, in-vehicle noise is not significantly different from standard noise. This indicates that in vehicle detection and prediction of cardiac events is possible.

\section{Conclusion}

This study has reinforced that simple signal processing methods, such as HR and HRV extraction, are successful on invehicle ECG data. Additional in-vehicle noise can mostly be attributed to the motion of the vehicle; for example electric motors do not contribute an overwhelming amount of noise to the raw signal. To combat these noise differences, additional accelerometer data to record movements for the vehicle, as well as the driver, could be incorporated into data collection and greatly improve the ECG signal processing.

This work illustrates how physiological monitoring can increase driver safety, specifically when incorporated into the personal vehicles of at-risk drivers. We explained the impact that a cardiac monitoring system would have on travel safety, as well as the need for such a system. We addressed the unavoidable noise complications for certain physiological monitors, such as the ECG.

Preliminary data collection and noise difference analysis have been performed to analyze the accuracy of essential signal processing algorithms on in-vehicle ECG, as well as identify the frequency bands most impacted by in-vehicle noise. We conclude that the additional in-vehicle noise for cardiac monitoring systems should not be an insurmountable obstacle for an in-vehicle driver monitoring system.

We ultimately determine that it is completely feasible to perform in-vehicle detection of incapacitating severe cardiac events such as MI and other arrhythmias.

Future studies are currently being conducted to further progress in the area, including a larger version of this study and the development of highly accurate in-vehicle medical event detection and prediction algorithms. Additionally, ECG processing for cardiac event detection can be performed as long as a signal is accurate enough. Therefore, collecting ECG with a mobile monitor maintaining high accuracy of detection is necessary. There is an option to explore alternative ECG collection devices such as implanted pacemakers, ECG watches, or other monitors worn on the extremities. The signal provided by these devices could be compatible with an updated version of most working ECG processing models. In addition, in-seat ECG collection devices could potentially be used for non-contact event detection, given that a high quality signal can be provided by the sensors.

This paper is written based on a proceeding presented at JSAE FAST-zero'17 Meeting.

\section{References}

(1) T.A. Zografos, and D.G. Katritsis: Guidelines and regulations for driving in heart: Hellenic J Cardiol, Vol. 51, No. 3, pp.226-234. (2010).

(2) P.C. Yang, J.H. Cheng, M.S. Tu, C.H. Tseng: A SmartphoneBased Heart Rate Variability Analysis System for Vehicle Drivers: IEEE International Conference on ITS Telecommunications (ITST), pp.827-831 (2012)

(3) R. Roy and K. Venkatasubramanian: EKG/ECG Based Driver Alert System for Long Haul Drive: Indian journal of science and Technology, Vol. 8, No. 19, pp.1-6 (2015).

(4) S. Hu, R.L. Bowlds, Y. Gu, and X. Yu: Pulse Wave Sensor for Non-Intrusive Driver's Drowsiness Detection: Annual International Conference of the IEEE Engineering in Medicine and Biology Society, pp.2312-2315 (2009).

(5) B. Chamadiya, S. Heuer, U.G. Hofmann, and M. Wagner: Towards a Capacitively Coupled Electrocardiography System for Car Seat Integration: 4th European Conference of the International Federation for Medical and Biological Engineering, pp.1217-1221. (2009).

(6) S. Leonhardt and A. Aleksandrowicz: Non-contact ECG Monitoring for Automotive Application: 5th International Summer School and Symposium on Medical Devices and Biosensors, ISSS- MDBS 2008, pp. 183-185 (2008).

(7) J. Schneider, C. Koellner, and S. Heuer: An Approach to Automotive ECG Measurement Validation Using a CarIntegrated Test Framework: IEEE Intelligent Vehicles Symposium (IV), pp.950-955 (2012).

(8) T. Wartzek, B. Eilebrecht, J. Lem, H.J. Lindner, S. Leonhardt, and M. Walter: ECG on the Road: Robust and Unobtrusive Estimation of Heart Rate: IEEE Transactions on Biomedical Engineering, Vol. 58, No. 11, pp.3112-3120 (2011).

(9) G.D. Clifford, F. Azuaje, and P. McSharry: ECG Statistics, 
Noise, Artifacts, And Missing Data: Advanced Methods and Tools for ECG Data Analysis, pp.6-18 (2006).

(10) G.M. Friesen, T.C. Jannett, M.A. Jadallah, S.L. Yates, S.R Quint, and H.T. Nagle: A Comparison of the Noise Sensitivity of Nine QRS Detection Algorithms: IEEE Transactions on biomedical engineering, Vol. 37, No. 1, pp.85-98, (1990).

(11) A. Baranchuk, J. Kang, C. Shaw, D. Campbell, S. Ribas, W.M. Hopman, H. Alanazi, D.P. Redfearn, and C.S. Simpson: Electromagnetic Interference of Communication Devices on ECG Machines: Clinical cardiology, Vol. 32, No. 10, pp.588592 (2009).

(12) A.L. Goldberger, L.A.N Amaral, G.L. Hausdorff, P.C, et al.: PhysioBank, PhysioToolkit, and PhysioNet: Components of a New Research Resource for Complex Physiologic Signals: Circulation, Vol. 101, No. 23, pp.215-220 (2000).

(13) R. Bousseljot, D. Kreiseler, and A. Schnabel: Nutzung der EKG-Signaldatenbank CARDIODAT der PTB über das
Internet: Biomedizinische Technik/Biomedical Engineering 40.s1, pp.317-318 (1995).

(14) A. Taddei, G. Distante, M. Emdin, P. Pisani, G. B. Moody, C. Zeelenberg, and C. Marchesi: The European ST-T Database: Standard for Evaluating Systems for the Analysis of ST-T Changes in Ambulatory Electrocardiography.: European heart journal, Vol. 13, No. 9, pp.1164-1172 (1992).

(15) G.B. Moody, and G.M. Roger: The Impact of the MIT-BIH Arrhythmia Database: IEEE Engineering in Medicine and Biology Magazine Vol.20, No.3, pp.45-50 (2001).

(16) F. Jager, A. Taddei, G.B. Moody, M. Emdin, G. Antolic, R. Dorn, A. Smrdel, C. Marchesi, and R.G. Mark: Long-term ST Database: A Reference for the Development and Evaluation of Automated Ischaemia Detectors and for the Study of the Dynamics of Myocardial Ischaemia: Medical \& Biological Engineering \& Computing, Vol. 41, No. 2, pp.172-183 (2003).

(17) J. Pan, W.J. Tompkins: A real-time QRS detection algorithm: IEEE Trans. Biomed. Eng. Vol. 32, No. 3, pp. 230-236 (1985) 